\title{
Pre-Exercise Subjective Estimation of Heart Rate in Different Physical Activities among Physical Education Students
}

\author{
Yoav Meckel1, Aya Ekshtein ${ }^{1}$, Sharon Tsuk ${ }^{1}$, Alon Eliakim ${ }^{1,2}$ \\ ${ }^{1}$ The Academic College at Wingate, Wingate Institute, Wingate, Israel \\ ${ }^{2}$ Meir Medical Center, Sackler School of Medicine, Tel Aviv University, Tel Aviv, Israel \\ Email: meckel@wincol.ac.il
}

How to cite this paper: Meckel, Y., Ekshtein, A., Tsuk, S., \& Eliakim, A. (2019). Pre-Exercise Subjective Estimation of Heart Rate in Different Physical Activities among Physical Education Students. Advances in Physical Education, 9, 42-52. https://doi.org/10.4236/ape.2019.91004

Received: November 14, 2018

Accepted: January 6, 2019

Published: January 9, 2019

Copyright ( 2019 by author(s) and Scientific Research Publishing Inc. This work is licensed under the Creative Commons Attribution International License (CC BY 4.0).

http://creativecommons.org/licenses/by/4.0/

(c) (i) Open Access

\begin{abstract}
Physical education teachers should be able to evaluate exercise intensity objectively as well as subjectively in order to implement the appropriate load during activity sessions. Whereas measuring oxygen uptake or blood lactate level during exercise may be a complicated task in field conditions, monitoring heart rate $(\mathrm{HR})$ values can be a relatively simple procedure when performed outdoors. The aim of this study was to examine the subjective estimation accuracy of HR in different exercises among physical education students. Pre-exercise estimations of HR were collected from 180 students prior to a multi-task activity session. The estimated HR values were then compared with true HR values, which were monitored throughout the session using a portable HR monitor system. A mean significant difference of $13.4 \%$ was found between the estimated and actual HRs for all activities. A majority of the students (70\%) estimated HR values to be lower than the true values. The most accurate HR estimations were found in extremely easy or in extremely difficult activities, whereas the least accurate estimations were found in moderate-level activities. No significant differences were found in HR estimation accuracy between males and females or between individuals with higher and with lower aerobic fitness. The results indicated that physical education students can estimate their HR responses to a reasonable degree of accuracy, but with most underestimating them compared to the actual values.
\end{abstract}

\section{Keywords}

Aerobic Exercises, Training, Multi-Task Activity, Moderate Efforts

\section{Introduction}

Assessment of physical activity intensity is usually determined by monitoring 
standard physiological variables such as heart rate (HR), oxygen consumption $\left(\mathrm{VO}_{2}\right)$, and blood lactate level (see, for example, Achten \& Jeukendrup, 2003; Alexander et al., 2012; Kenney, Wilmore, \& Costill, 2015). Rating of perceived exertion (RPE) can also be used as a subjective variable that corresponds with objective measures of physiologic strain (see Borg, 1982). Nevertheless, despite its simplicity, this method may lack the accuracy usually required for measuring exercise intensity.

Whereas measuring $\mathrm{VO}_{2}$ uptake or blood lactate level during exercise may be a complicated task in outdoor conditions, monitoring HR values is a relatively simple procedure when using a portable HR monitor system outdoors. Indeed, it is documented that $\mathrm{HR}$ and $\mathrm{VO}_{2}$ are linearly related in trained and untrained individuals throughout the major portion of the exercise range (Borresen \& Lambert, 2008; Brown \& Brown, 2007). Therefore, monitoring HR responses is considered to be a practical and popular method for measuring exercise intensity level, especially in aerobic-type exercise. Aerobic exercise has previously defined as physical activity during which the intensity is easily sustained with little variability in HR responses (American College of Sports Medicine, 2017). However, it should be noted that some factors might interfere with or influence HR responses during physical activity. Among these factors are environmental conditions, emotions, previous food intake, body position, sex, age, muscle group exercised, if the exercise is continuous or intermittent, and whether the muscles act statically or dynamically (McArdle, Magel, \& Kyvallos, 1971; Meijer, Westerterp, Koper, \& ten Hoor, 1989). For example, HR in aerobic dance will exceed the HR during treadmill running at the same $\mathrm{VO}_{2}$ level (see, Parker, Hurley, Hanlon, \& Vaccaro, 1989). Consistent with this finding, higher HR was measured in upper-body exercise or when muscles act statically in straining-type exercise than in dynamic leg exercise at any sub-maximal level (Mostardi, Gandee, \& Norris, 1981; Rotstein \& Meckel, 2000). Consequently, applying HR during upper-body or static exercise to the $\mathrm{HR}-\mathrm{VO}_{2}$ curve line developed during running or cycling may over-predict the actual $\mathrm{VO}_{2}$ (Vokac, Bell, Bautz-Holter, \& Rodahl, 1975). In addition, HR responses to submaximal exercise are different between trained and untrained individuals. In this respect, HR for a given exercise load will be lower for trained compared to untrained individuals, mainly due to the higher stroke volume of trained individuals (Warburton et al., 2002).

Given the various factors involved and the variability in HR responses during physical activity, it seems that a measure of knowledge and personal experience is required before an individual will be able to reasonably estimate his/her HR responses to different types of exercise. This estimation may be valuable as a tool for prescribing or monitoring the exercise intensity level, particularly when no advanced technical means or devices are on hand. Such knowledge may be especially important for physical education teachers, who are responsible for teaching and instructing their pupils about the expected effort required in different physical tasks. Such knowledge may also be important for special population 
such as trained athletes or older people who are trying to reduce risk factors for coronary heart diseases through exercise (Karapetian, Engels, \& Gretebeck, 2008). These populations are required to exercise in specific intensities according to their needs and limitations (Bentley, Newell, \& Bishop, 2007). The purpose of the present study, therefore, was to examine the level of accuracy in the subjective estimation of HR in different exercises among physical education students. The level of estimation accuracy was also compared between genders, as well as between physical education students with different levels of aerobic fitness.

\section{Method}

\subsection{Participants}

One hundred and eighty students ( 98 women and 82 men, age $25.8 \pm 3.4$ yrs) attending a college for physical education and sport sciences participated in the study. The participants were in the third year of a four-year academic program and had taken numerous relevant theoretical and practical classes, such as general physiology, exercise physiology, physical fitness, and conditioning. The students' typical individual weekly physical activity schedule included $1-2$ aerobic training sessions and 2 - 3 ball-game activity sessions, lasting 30 and $60 \mathrm{~min}$ each, respectively. The study was approved by the Institution's ethical committee, and a signed informed consent was obtained from all the participants.

\subsection{Measurements}

Data collection was performed during two main events: (a) a multi-task activity session, and (b) a $5000 \mathrm{~m}$ run session.

1) The multi-task activity session. The multi-task activity session included a series of 14 activities that were performed consecutively and lasted a total time of about $60 \mathrm{~min}$. The list of activities in order of performance, together with the time span for each, is presented in Table 1 . The list of activities was presented to the participants prior to the beginning of the session, and they were asked to write down their estimated personal peak HR for each of the 14 upcoming activities.

The participants were familiar with the activities, having routinely performed them (with no monitoring system) during different practical sports classes. The participants were free to ask questions regarding the activities before giving their estimations of their HR values. Upon completion of the HR estimation process, the participants were asked to put on a Polar HR monitor system (Polar Accurex Plus, Polar Electro, Woodbury, NY, USA) using a chest strap, in addition to a wristwatch. Holding a pencil and a page with the list of activities, the participants then started to perform the 14 activities as they appeared and were described on the list, while the HR monitor system recorded their actual HR for each activity. Throughout the session, the participants wrote down their actual HR response upon completion of each of the 14 activities. The activities of this 
Table 1. Description and time span of the 14 activities included in the multi-task activity session.

\begin{tabular}{cc}
\hline Description of Activity & Time (min) \\
\hline Pre-exercise rest & 1 \\
Warm-up-slow-pace jogging & 8 \\
Stretching and flexibility drills & 3 \\
Upper-body power drills (push-ups and sit-ups) & 5 \\
Recovery walk & 3 \\
Continuous moderate-pace jogging & 12 \\
Strides-a 150 m accelerated run & 0.5 \\
Recovery walk & 1 \\
Strides-a 150 m accelerated run & 0.5 \\
Recovery walk & 1 \\
300 m uphill running & 2 \\
Recovery walk & 5 \\
Continuous moderate-pace jogging & 12 \\
Recovery walk & 3 \\
Total & 57 \\
\hline
\end{tabular}

session were performed in an open-space park located near the campus, where all the drills could be performed as required. Upon completion of the session, the participants could analyse and compare their pre-exercise estimated HR values to the actual recorded HR values. The mean estimated and the actual HR values for all the activities appear in Table 2 .

2) The $5000 \mathrm{~m}$ run session. The $5000 \mathrm{~m}$ run was performed about a week after the multi-task session. This run was performed in order to determine the participants' aerobic fitness category level. For the male students, a running time faster than 22 min was considered high aerobic fitness, whereas a running time slower than 27 min was considered low aerobic fitness. The equivalent running time categories for female students was $28 \mathrm{~min}$ or faster for high aerobic fitness, and $33 \mathrm{~min}$ or slower for low aerobic fitness. The run was performed on a flat field track surrounding the campus. Running times were taken by hand using a standard stopwatch, and were rounded off to the nearest $0.1 \mathrm{sec}$.

Both events-the multi-task session and the $5000 \mathrm{~m}$ run-were performed in homogenous sub-groups of 20 - 25 female or male participants, with similar comfortable environmental conditions (time: 8:30 a.m.; temperature: $20^{\circ} \mathrm{C}$ $22^{\circ} \mathrm{C}$; wind: $0.1-0.3 \mathrm{~m} / \mathrm{sec}$; humidity: $40 \%-50 \%$ ) for all groups. In order to eliminate unnecessary fatigue symptoms, the participants were instructed to avoid any intense physical activity for 48 hrs prior to each of the two events. In addition, in order to minimize unnecessary effects on their HR, the participants were instructed to stop eating or consuming any caffeine at least $90 \mathrm{~min}$ before the multi-task activity and the $5000 \mathrm{~m}$ run. 
Table 2. Absolute differences between estimated and actual heart rate (mean \pm SD) for each the 14 activities and for the mean in the multi-task session.

\begin{tabular}{lccc}
\hline \multicolumn{1}{c}{ Activity } & $\begin{array}{c}\text { Estimated HR } \\
(\mathrm{b} / \mathrm{min})\end{array}$ & $\begin{array}{c}\text { Actual HR } \\
(\mathrm{b} / \mathrm{min})\end{array}$ & $\begin{array}{c}\text { Differences } \\
(\%)\end{array}$ \\
\hline 1) Rest & $72 \pm 11$ & $77 \pm 12.5^{*}$ & $9.4 \pm 8.6$ \\
2) Warm-up jog & $126 \pm 23.6$ & $151 \pm 23.7^{*}$ & $18.1 \pm 14.0$ \\
3) Stretching & $97 \pm 17.1$ & $109 \pm 18.5^{*}$ & $14.7 \pm 12.1$ \\
4) Power drills & $122 \pm 20.7$ & $142 \pm 20.1^{*}$ & $16.5 \pm 11.6$ \\
5) Recovery walk & $102 \pm 19.1$ & $117 \pm 20.6^{*}$ & $15.2 \pm 11.8$ \\
6) Jogging & $136 \pm 23.3$ & $163 \pm 19.7^{*}$ & $17.1 \pm 11.7$ \\
7) 150 m strides & $158 \pm 20.2$ & $167 \pm 20.7^{*}$ & $10.3 \pm 10.6$ \\
8) Recovery walk & $121 \pm 22.7$ & $133 \pm 22.8^{*}$ & $13.8 \pm 11.5$ \\
9. 150 m strides & $162 \pm 19.8$ & $170 \pm 15.8^{*}$ & $9.1 \pm 8.5$ \\
10. Recovery walk & $126 \pm 22.2$ & $135 \pm 22.1^{*}$ & $12.8 \pm 12.6$ \\
11. Uphill running & $174 \pm 19.4$ & $179 \pm 18.8^{*}$ & $8.2 \pm 9.8$ \\
12. Recovery walk & $126 \pm 23.8$ & $124 \pm 21.5$ & $12.9 \pm 11.4$ \\
13. Jogging & $141 \pm 23.0$ & $164 \pm 21.5^{*}$ & $15.6 \pm 11.3$ \\
14. Recovery walk & $111 \pm 21.9$ & $122 \pm 20.1^{*}$ & $14.8 \pm 13.3$ \\
$\quad---$ & ---- & $13.4 \pm 6.3$ \\
\hline Mean & & &
\end{tabular}

${ }^{*} p<0.001$.

\subsection{Statistical Analysis}

A one-sample t-test was performed to determine absolute (deviation from zero state) differences between the estimated and actual HR for each activity (Table 2). The estimation status for each activity was recoded into three categories, as follows: underestimation, overestimation, and accurate estimation. The frequencies of estimation for these categories appear in Table 3. An independent t-test, with Cohen's d effect size, was performed for each activity in order to compare the female and male students' absolute estimation accuracy. Similarly, an independent $\mathrm{t}$-test was performed for each activity in order to compare absolute estimation accuracy in participants with high and low aerobic fitness. Significance level was set at $p \leq 0.05$.

\section{Results}

A description of the 14 activities in the multi-task session, and the time span for each activity, are presented in Table 1. As can be seen, the list of activities contains different type of exercises-aerobic as well as anaerobic in nature-with different performance time for each activity. Table 2 presents the estimated and actual HR and absolute differences for each of the 14 activities in the multi-task session. The highest actual HR responses were registered in uphill running (179 $\mathrm{b} / \mathrm{min}$ ) and in $150 \mathrm{~m}$ strides $(170 \mathrm{~b} / \mathrm{min})$, while the lowest actual HR responses were registered in recovery walk $(117 \mathrm{~b} / \mathrm{min})$ and in stretching exercise (109 $\mathrm{b} / \mathrm{min})$. Values were calculated for each participant as the absolute values of the 
Table 3. Overestimation, accurate and underestimation percentage (mean \pm SD) from actual heart rate for each activity.

\begin{tabular}{|c|c|c|c|c|c|c|}
\hline \multirow[t]{2}{*}{ Activity } & \multicolumn{2}{|c|}{ Estimation Status } & \multicolumn{4}{|c|}{ Mean \pm SD } \\
\hline & Underestimation & $64.6 \%$ & - & 11.4 & \pm & 7.9 \\
\hline \multirow[t]{3}{*}{ 1) Rest } & Accurate estimation & $12.6 \%$ & & 0 & \pm & 0 \\
\hline & Overestimation & $22.9 \%$ & + & 8.9 & \pm & 9.3 \\
\hline & Underestimation & $87.0 \%$ & - & 19.3 & \pm & 13.2 \\
\hline \multirow[t]{3}{*}{ 2) Warm-up jog } & Accurate estimation & $2.3 \%$ & & 0 & \pm & 0 \\
\hline & Overestimation & $10.7 \%$ & + & 12.3 & \pm & 17.6 \\
\hline & Underestimation & $74.2 \%$ & - & 16.5 & \pm & 11.3 \\
\hline \multirow[t]{3}{*}{ 3) Stretching } & Accurate estimation & $6.7 \%$ & & 0 & \pm & 0 \\
\hline & Over estimation & $19.1 \%$ & + & 12.9 & \pm & 13.5 \\
\hline & Under estimation & $80.8 \%$ & - & 18.4 & \pm & 11.3 \\
\hline \multirow[t]{3}{*}{ 4) Power drills } & Accurate estimation & $3.4 \%$ & & 0 & \pm & 0 \\
\hline & Overestimation & $15.8 \%$ & + & 10.7 & \pm & 9.4 \\
\hline & Underestimation & $80.5 \%$ & - & 16.6 & \pm & 11.6 \\
\hline \multirow[t]{3}{*}{ 5) Recovery walk } & Accurate estimation & $1.7 \%$ & & 0 & \pm & 0 \\
\hline & Overestimation & $17.8 \%$ & + & 10.5 & \pm & 11.3 \\
\hline & Underestimation & $88.8 \%$ & - & 18.7 & \pm & 11.2 \\
\hline \multirow[t]{3}{*}{ 6) Jogging } & Accurate estimation & $4.5 \%$ & & 0 & \pm & 0 \\
\hline & Overestimation & $6.7 \%$ & + & 6.5 & \pm & 5.9 \\
\hline & Underestimation & $68.6 \%$ & - & 11.1 & \pm & 9.6 \\
\hline \multirow[t]{3}{*}{ 7) $150 \mathrm{~m}$ strides } & Accurate estimation & $4.6 \%$ & & 0 & \pm & 0 \\
\hline & Overestimation & $26.9 \%$ & + & 9.8 & \pm & 12.9 \\
\hline & Underestimation & $68.6 \%$ & - & 15.3 & \pm & 10.9 \\
\hline \multirow[t]{3}{*}{ 8) Recovery walk } & Accurate estimation & $4.7 \%$ & & 0 & \pm & 0 \\
\hline & Overestimation & $26.7 \%$ & + & 12.3 & \pm & 12.1 \\
\hline & Underestimation & $62.9 \%$ & - & 10.6 & \pm & 8.5 \\
\hline \multirow[t]{3}{*}{ 9) $150 \mathrm{~m}$ strides } & Accurate estimation & $3.8 \%$ & & 0 & \pm & 0 \\
\hline & Overestimation & $33.3 \%$ & + & 7.2 & \pm & 7.9 \\
\hline & Underestimation & $60.4 \%$ & - & 15.1 & \pm & 10.8 \\
\hline \multirow[t]{3}{*}{ 10) Recovery walk } & Accurate estimation & $6.3 \%$ & & 0 & \pm & 0 \\
\hline & Overestimation & $33.3 \%$ & + & 11.2 & \pm & 14.9 \\
\hline & Underestimation & $56.9 \%$ & - & 9.7 & \pm & 8.4 \\
\hline \multirow[t]{3}{*}{ 11) Uphill running } & Accurate estimation & $6.3 \%$ & & 0 & \pm & 0 \\
\hline & Overestimation & $36.8 \%$ & + & 7.2 & \pm & 11.8 \\
\hline & Underestimation & $43.9 \%$ & - & 11.6 & \pm & 8.5 \\
\hline \multirow[t]{3}{*}{ 12) Recovery walk } & Accurate estimation & $3.5 \%$ & & 0 & \pm & 0 \\
\hline & Overestimation & $52.6 \%$ & + & 14.9 & \pm & 13.2 \\
\hline & Underestimation & $81.3 \%$ & - & 17.5 & \pm & 10.9 \\
\hline \multirow[t]{3}{*}{ 13) Jogging } & Accurate estimation & $4.1 \%$ & & 0 & \pm & 0 \\
\hline & Overestimation & $14.6 \%$ & + & 8.8 & \pm & 9.4 \\
\hline & Underestimation & $66.9 \%$ & - & 16.4 & \pm & 11.1 \\
\hline \multirow[t]{3}{*}{ 14) Recovery walk } & Accurate estimation & $8.6 \%$ & & 0 & \pm & 0 \\
\hline & Overestimation & $24.6 \%$ & + & 15.5 & \pm & 17.6 \\
\hline & Underestimation & $70.4 \%$ & - & 15.3 & \pm & 10.37 \\
\hline \multirow[t]{2}{*}{ TOTAL } & Accurate estimation & $5.2 \%$ & & 0 & \pm & 0 \\
\hline & Overestimation & $24.4 \%$ & + & 10.6 & \pm & 11.91 \\
\hline
\end{tabular}


differences between predicted and actual HR. A mean significant difference of 13.4\% was found between the estimated and actual HRs for all activities.

Table 3 presents overestimation, accurate estimation, and underestimation (Mean \pm SD) from the actual HR rate for each activity. Most of the students (70\%) estimated HR responses to be lower than the true values. The highest underestimation value $(88 \%)$ was noticed in recovery walk.

Differences between actual and estimated HR for the female and male students in each activity are presented in Table 4. No significant differences (with the exception of stretching exercises and the recovery walk) were found for mean deviation between estimated and actual HR. Differences between actual and estimated HR for participants with high and low aerobic fitness in each activity are presented in Table 5. No significant differences (with the exception of warm-up and stretching exercises) were found for mean deviation of estimated HR from the actual HR between participants with high and with low aerobic fitness.

\section{Discussion}

The main finding of the present study was that there were significant differences between the estimated and actual HRs in 13 out of the 14 activities performed by the third-year physical education students.

\subsection{Differences in Estimation Accuracy between the Different Activities}

The mean deviation of the estimated HR from the actual HR for all the activities

Table 4. Absolute differences between actual and estimated heart rate (mean $\pm \mathrm{SD}$ ) for females and males.

\begin{tabular}{lccc}
\hline \multirow{1}{*}{ Activity } & \multicolumn{3}{c}{ Differences } \\
\cline { 2 - 4 } & Females $(\mathrm{n}=98)$ & Males $(\mathrm{n}=82)$ & Cohen's d \\
\hline 1) Rest & $9.5 \pm 9.2$ & $9.3 \pm 8.0$ & 0.02 \\
2) Warm-up jog & $19.7 \pm 14.8$ & $16.3 \pm 12.8$ & 0.24 \\
3) Stretching & $16.7 \pm 12.3$ & $12.2 \pm 11.5^{\star}$ & 0.37 \\
4) Power drills & $16.9 \pm 12.4$ & $16.0 \pm 10.7$ & 0.08 \\
5) Recovery walk & $17.0 \pm 12.6$ & $13.0 \pm 10.3^{\star}$ & 0.35 \\
6) Jogging & $17.4 \pm 12.3$ & $16.7 \pm 11.0$ & 0.06 \\
7) 150 m strides & $10.8 \pm 11.2$ & $9.7 \pm 9.9$ & 0.10 \\
8) Recovery walk & $12.9 \pm 10.1$ & $14.9 \pm 12.8$ & 0.18 \\
9) 150 m strides & $9.0 \pm 8.5$ & $9.1 \pm 8.5$ & 0.01 \\
10) Recovery walk & $14.1 \pm 13.8$ & $11.4 \pm 11.0$ & 0.22 \\
11) Uphill running & $7.3 \pm 10.9$ & $9.3 \pm 8.3$ & 0.21 \\
12) Recovery walk & $12.9 \pm 11.7$ & $12.9 \pm 11.2$ & 0.00 \\
13) Jogging & $15.5 \pm 11.9$ & $15.6 \pm 10.7$ & 0.01 \\
14) Recovery walk & $15.3 \pm 13.5$ & $14.2 \pm 13.1$ & 0.09 \\
\multicolumn{1}{c}{ Total } & $13.8 \pm 6.5$ & $13.0 \pm 6.1$ & 0.13 \\
\hline
\end{tabular}

${ }^{\star} p<0.05$. 
Table 5. Absolute differences between actual and estimated heart rate (mean \pm SD) for participants with high and low aerobic fitness.

\begin{tabular}{lccc}
\hline \multirow{2}{*}{ Activity } & \multicolumn{3}{c}{ Differences } \\
\cline { 2 - 4 } & High $(\mathrm{n}=83)$ & Low $(\mathrm{n}=97)$ & Cohen's d \\
\hline 1) Rest & $10.1 \pm 9.8$ & $10.0 \pm 8.1$ & 0.01 \\
2) Warm-up jog & $15.9 \pm 12.8$ & $21.4 \pm 14.6^{*}$ & 0.40 \\
3) Stretching & $12.9 \pm 10.7$ & $18.1 \pm 13.8^{*}$ & 0.43 \\
4) Power drills & $15.3 \pm 11.9$ & $17.6 \pm 10.4$ & 0.21 \\
5) Recovery walk & $14.2 \pm 10.5$ & $18.0 \pm 13.0$ & 0.32 \\
6) Jogging & $15.7 \pm 11.4$ & $19.2 \pm 11.9$ & 0.30 \\
7) 150 m strides & $9.6 \pm 11.8$ & $10.7 \pm 8.7$ & 0.10 \\
8) Recovery walk & $14.2 \pm 11.6$ & $14.5 \pm 11.7$ & 0.03 \\
9) 150 m strides & $7.9 \pm 8.2$ & $9.1 \pm 7.4$ & 0.15 \\
10) Recovery walk & $14.6 \pm 15.7$ & $11.7 \pm 10.2$ & 0.23 \\
11) Uphill running & $7.6 \pm 8.4$ & $7.4 \pm 6.7$ & 0.02 \\
12) Recovery walk & $12.8 \pm 12.3$ & $13.2 \pm 11.1$ & 0.03 \\
13) Jogging & $16.1 \pm 12.0$ & $16.0 \pm 11.0$ & 0.01 \\
14) Recovery walk & $14.5 \pm 11.9$ & $15.8 \pm 13.5$ & 0.10 \\
Total & $12.9 \pm 6.0$ & $14.5 \pm 6.0$ & 0.27 \\
\hline
\end{tabular}

${ }^{*} p<0.05$.

was $13.4 \%$ (see Table 2). It was also found that the most accurate HR estimations were in the two extremes of the activities-the hardest and the easiest ones. As such, the deviations for uphill running and $150 \mathrm{~m}$ strides, the most intense activities, were $8.2 \%$ and $9.1 \%$, respectively, and for rest and the recovery walk, representing the easiest activities, the deviations were $9.4 \%$ and $12.9 \%$, respectively.

The greatest differences between the estimated and actual HRs were found in moderate-level activities, such as jogging-17.1\% - 18.1\%. These findings may demonstrate that physical education students are more familiar with the boundaries of their HR responses, possibly because they have experienced these boundaries before and can relate to them during the relevant intense or light-level activities. In contrast, it seems that the wide range of possible sub-maximal HRs during moderate-level activities makes accurate HR estimation a difficult task. It may also indicate that people are able to recognize and recall the sensations of extreme experiences (such as maximal level efforts) better than those of conventional experiences (such as sub-maximal level efforts) (Wiener, Garber, \& Manfredi, 1995).

In spite of the significant differences that were found between the estimated and actual HRs for all the activities in the multi-task session, any attempt to categorize these values in to levels of accuracy would be entirely arbitrary, since to the best of our knowledge no other research has examined the accuracy of HR estimations and compared it to the actual HR in this population. Nevertheless, in 
our college we use the following deviation values to grade the level of HR estimation accuracy among the students in fitness classes: $<5 \%$-very good, 5\% $10 \%$-good, $10 \%$ - 15\%-reasonable, $15 \%<$ - do not know your body sufficiently. Using this grading scale, our present sample attained an average score of "reasonable" for the different activities. In order to obtain a better perspective and to appreciate the level of accuracy for HR estimation, future studies should examine non-active or highly trained populations.

\subsection{Underestimation of HR in Most Activities}

The data in Table 3 reveal that the majority of the physical education students estimated HR responses to be lower than their actual values in most of the activities. Specifically, a mean value of $70.4 \%$ of the students underestimated the HR responses by $15.3 \%$, whereas a mean of only $24.4 \%$ of the students overestimated HR by $10.6 \%$ of the actual value. These findings suggest that relatively active individuals, such as physical education students, perceive physical efforts (which in the present study were mostly leisure-type) as easier than they actually are.

One specific reason for the HR underestimation among the students in the present study may be the manner in which the activities were performed in the multi-task session. It is possible that although the students were informed about the consecutive manner of the activities in the session, they perceived and related to each of them as a single and isolated activity, ignoring the possible accumulative fatigue that developed throughout the session. It is also possible that the non-stressed academic setting of the session in the present study, rather than a stressed athletic or competitive setting (Yamaji \& Shephard, 1986), caused the participants to feel calm and secure, leading to under estimated HR responses. In order to assess this assumption and to understand its broader applications more extensively, it would be interesting to compare the individuals' rate of perceived exertion (RPE) estimation (Borg, 1982) prior to the execution of a multi-task session with the actual measurements during the session. As noted earlier, it is suggested that future studies examine non-active or highly trained populations as well.

\subsection{Sub-Groups Observation}

Another interesting aspect of the present study was the observation of specific sub-groups among the present sample. For this purpose, a separation was made between female students and male students and between individuals with a high and with a low aerobic fitness level.

When comparing female students to male students, no significant differences were found for the mean deviation between estimated and actual HR, with the exception of stretching exercises and the recovery walk, where male students were significantly more accurate than female students (Table 4). In addition, no significant differences were found for mean deviation of estimated HR from the 
actual HR between participants with high and with low aerobic fitness, with the exception of warm-up and stretching exercises, where participants with high aerobic fitness were significantly more accurate than participants with a lower aerobic fitness level (Table 5).

These findings are somewhat surprising, since one might expect aerobically fit individuals, who experience diverse levels of physical effort in their training, to better recognize and appreciate the levels of physical difficulties and their corresponding HRs in different types of exercise, compared to relatively unfit and inexperienced individuals. However, it is possible that the population of physical education students in our study presented a relatively narrow range of fitness levels (meaning that even the participants with a "low" fitness level were relatively fit compared to the general population). Consequently, there were no differences between the relatively fit students with higher fitness and those with lower aerobic fitness. It is possible that in order to detect significant differences in estimated HR for a given exercise, greater differences in fitness should exist between the two sub-groups. Moreover, the findings may point out the importance of using a portable HR monitoring device (such as the Polar monitoring system) as a tool for assessing exercise intensity, not only among untrained individuals but among the relatively trained as well (Achten, \& Jeukendrup, 2003; Alexander et al., 2012).

\section{Conclusion}

It seems that physical education students can estimate HR responses to a reasonable degree of accuracy for different types of exercises. However, these students can more accurately estimate HR responses for the two extremes of activities (i.e., the hardest and easiest activities) than for moderate intensity-level activities. It is also apparent that most physical education students estimate their HR responses to be lower than their actual values for different physical activities.

In addition, in most cases there were no differences in HR estimation accuracy between males and females or between individuals with higher and with lower aerobic fitness. This may demonstrate the importance of using HR monitoring devices during physical activity for all kinds of populations-trained as well as untrained. Lastly, it appears that in order to obtain a better perspective and to appreciate the level of accuracy of HR estimation, future studies should examine non-active as well as highly trained populations.

\section{Conflicts of Interest}

The authors declare no conflicts of interest regarding the publication of this paper.

\section{References}

Achten, J., \& Jeukendrup, A. E. (2003). Heart Rate Monitoring: Applications and Limitations. Sports Medicine, 33, 517-538. https://doi.org/10.2165/00007256-200333070-00004 
Alexander, D., da Silva, C. D., Hill-Haas, S., Wong, del P., Natali, A. J., De Lima, J. R.,... Karim, C. (2012). Heart rate monitoring in soccer: interest and limits during competitive match play and training, practical application. Journal of Strength and Conditioning Research, 26, 2890-2906. https://doi.org/10.1519/JSC.0b013e3182429ac7

American College of Sports Medicine (2017). ACSM's Guidelines for Exercise Testing and Prescription (10th ed.). Wolters Kluwer.

Bentley, D. J., Newell, J., \& Bishop D. (2007). Incremental Exercise Test Design and Analysis: Implications for Performance Diagnostics in Endurance Athletes. Sports Medicine, 37, 575-586. https://doi.org/10.2165/00007256-200737070-00002

Borg, G. A. (1982). Psychophysical Bases of Perceived Exertion. Medicine \& Science in Sports \& Exercise, 14, 377-381. https://doi.org/10.1249/00005768-198205000-00012

Borresen, J., \& Lambert, M. I. (2008). Autonomic Control of Heart Rate during and after Exercise: Measurements and Implications for Monitoring Training Status. Sports Medicine, 38, 633-646. https://doi.org/10.2165/00007256-200838080-00002

Brown, S. J., \& Brown, J. A. (2007). Resting and Postexercise Cardiac Autonomic Control in Trained Masters Athletes. The Journal of Physiological Sciences, 57, 23-29. https://doi.org/10.2170/physiolsci.RP012306

Karapetian, G. K., Engels, H. J., \& Gretebeck, R. J. (2008). Use of Heart Rate Variability to Estimate LT and VT. International Journal of Sports Medicine, 29, 652-657. https://doi.org/10.1055/s-2007-989423

Kenney, W. L., Wilmore, J. H., \& Costill, D. L. (2015). Physiology of Sport and Exercise (6th ed.). Champaign, IL: Human Kinetics.

McArdle, W. D., Magel, J. R., \& Kyvallos, L. C. (1971). Aerobic Capacity, Heart Rate and Estimated Energy Cost during Women's Competitive Basketball. Research Quarterly, 42, 178-186.

Meijer, G. A., Westerterp, K.R., Koper, H., \& ten Hoor, F. (1989). Assessment of Energy Expenditure by Recording Heart Rate and Body Acceleration. Medicine \& Science in Sports \& Exercise, 21, 343-347. https://doi.org/10.1249/00005768-198906000-00020

Mostardi, R. A., Gandee, R. N., \& Norris, W. A. (1981). Exercise Training Using Arms and Legs Versus Legs Alone. Archives of Physical Medicine and Rehabilitation, 62, 332-335.

Parker, S., Hurley, B. F., Hanlon, D.P., \& Vaccaro, P. (1989). Failure of Target Heart Rate to Accurately Monitor Intensity during Aerobic Dance. Medicine \& Science in Sports \& Exercise, 21, 230-234. https://doi.org/10.1249/00005768-198904000-00017

Rotstein, A., \& Meckel, Y. (2000). Estimation of \%VO2 Reserve from Heart Rate during arm Exercise and Running. European Journal of Applied Physiology, 83, 545-550. https://doi.org/10.1007/s004210000308

Vokac, Z., Bell, H., Bautz-Holter, E., \& Rodahl, K. (1975). Oxygen Uptake/Heart Rate Relationship in Leg and Arm Exercise, Sitting and Standing. Journal of Applied Physiology, 39, 54-59. https://doi.org/10.1152/jappl.1975.39.1.54

Warburton, D. E. R., Haykowsky, M. J., Quinney, H. A., Blackmore, D., Teo, K. K., \& Humen, D. P. (2002). Myocardial Response to Incremental Exercise in Endurance-Trained Athletes: Influence of Heart Rate, Contractility and the Frank-Starling Effect. Experimental Physiology, 87, 613-622. https://doi.org/10.1113/eph8702372

Wiener, S. P., Garber, C. E., \& Manfredi, T. G. (1995). A Comparison of Exercise Performance on Bicycle and Rowing Ergometers in Female Master Recreational Rowers. Journal of Sports Medicine and Physical Fitness, 35, 176-180.

Yamaji, K., \& Shephard, R. J. (1986). Heart Rate Overshoot in Running Events. British Journal of Sports Medicine, 20, 62-65. https://doi.org/10.1136/bjsm.20.2.62 